\section{Molecular Imaging in Oncology}

\author{
O. Schober and B. Riemann, Eds.
}

Heidelberg, Germany: Springer, 2013, 415 pages, $\$ 209$

Molecular imaging is defined as the visualization, characterization, and quantification of biologic processes at the cellular and molecular levels in living organisms. Molecular imaging allows the study of cellular and molecular pathways and disease mechanisms in their own physiologically authentic environment so that the molecular abnormalities that form the basis of the disease can be revealed. The process of molecular imaging includes the identification of key biotargets and the design and preclinical evaluation of specific probes. A multitude of such innovative probes has already entered clinical diagnostics in oncology. The impact of molecular imaging on diagnostics, therapy, and follow-up in cancer patients has been increasing significantly. There is no doubt that in future the emphasis will be on multimodality imaging in which morphologic, functional, and metabolic imaging techniques are combined in a single clinical investigation that will optimize diagnostic processes.

Among the increasing number of publications comprising all fields of molecular imaging, this handbook focuses on the growing impact of molecular imaging in oncology and addresses topics ranging from basic research to clinical applications in the era of evidence-based medicine. The editors carefully selected experts in the different fields of molecular imaging to outline the major trends and challenges of molecular imaging in oncology, bridging the gap between basic research and clinical applications in a unique way. This book is organized into 4 parts with 14 chapters. Part $\mathrm{I}$, with 5 chapters, is devoted to technology and probe design and examines CT, MR imaging, SPECT, ${ }^{18}$ F-labeled PET tracers, optical and optoacoustic imaging, and multifunctional MR imaging probes. Part II, with 4 chapters, discusses preclinical studies in detail, with particular attention on multimodality imaging such as PET/MR imaging. Part III, with 4 chapters, presents diverse clinical applications of molecular imaging in oncology. The final part, IV, deals with future challenges of multimodality imaging. The information is current, and the figures are clear and helpful.

Molecular imaging in oncology in the 21st century is not possible without close interdisciplinary and interfaculty collaborations, and this book highlights the potential reintegration of different disciplines. I highly recommend this book to nuclear physicians, radiologists, oncologists, chemists, physicists, mathematicians, and computer scientists.

\section{E. Edmund Kim}

University of California at Irvine

101 The City Dr. S.

Orange, $C A 92868$

E-mail: edmundek@uci.edu

Published online Apr. 4, 2013

DOI: 10.2967/jnumed.113.123109

\section{Imaging Tumor Response to Therapy}

\author{
M. Aglietta and D. Regge, Eds.
}

New York, NY: Springer, 2012, 174 pages, $\$ 139$

Cancer is a major human health problem, and a completely effective therapy is not available for most types of cancer. The expected benefit of the treatment is assessed in terms of a reduction in both the size of the tumor and dissemination of the cancer. Imaging is an ideal tool with which to evaluate response. The aim of using imaging as a surrogate biomarker for therapeutic response in oncology is to obtain a measure of disease extent and to better understand tumor response earlier than is possible with other biomarkers or with primary endpoints. When targeted therapy is available and can stabilize the disease, patient survival or quality of life improves to a greater extent than is possible with the transient tumor shrinkage induced by chemotherapy. There is evidence that tumor growth is strongly inhibited even if conventional imaging shows no change in tumor volume or a paradoxic increase. Therefore, in clinical practice the decision of whether to consider a treatment effective may be challenging. These problems are amplified during drug development, and improved patient survival is inappropriate in early clinical trials. Thus, surrogate endpoints of activity have become crucial. This book addresses these problems, and the detailed discussion of specific clinical situations contributes to and enhances the ongoing debate although it is not solved.

This relatively small book is organized into 3 parts with 9 chapters. Part I begins with the methodologic bases and discusses surrogate endpoints of clinical benefit and response to treatment, along with the role of imaging. Part II deals with the assessment of therapeutic response in metastatic disease from gastrointestinal stromal tumors, kidney cancer, and colon cancer. Part III covers the assessment of therapeutic response in locally advanced breast, lung, pancreas, and liver cancer. The book's multidisciplinary perspective well addresses the assessment of therapeutic response. Finding more reliable and reproducible measures of tumor response is one of the most important and difficult challenges facing modern radiology, as it requires an entirely new approach to imaging. The figures are clear and helpful, and the references are current. There is no index.

Considering that no increase is expected in the number of new therapies with strong evidence of a survival benefit entering the market, and that demands for health-care cost containment are becoming increasingly pressing, the role of nonvalidated surrogate endpoints should be carefully reconsidered, both in practice and in research. The choice of the primary endpoint should be based on sound biologic, pathophysiologic, and statistical evaluations to produce the best outcome for patients, as well as to determine validity, sensitivity to the study treatment, and feasibility. In the development of evidence-based practice guidelines, the quality of the available evidence should be carefully assessed, with greater attention placed on the outcomes considered in the specific recommendations. I highly recommend this book to radiologists, nuclear physicians, oncologists, and imaging scientists.

\section{E. Edmund Kim}

University of California at Irvine

101 The City Dr. S.

Orange, CA 92868

E-mail: edmundek@uci.edu 